\title{
Synergy between the Large Synoptic Survey Telescope and the Square Kilometre Array
}

\author{
David Bacon $^{* 1}$, Sarah Bridle ${ }^{2}$, Filipe B. Abdalla ${ }^{3,4}$, Michael Brown ${ }^{2}$, Philip Bull ${ }^{5}$, \\ Stefano Camera ${ }^{2}$, Rob Fender ${ }^{6}$, Keith Grainge ${ }^{2}$, Željko Ivezić ${ }^{7}$, Matt Jarvis ${ }^{6,8}$, Neal \\ Jackson $^{2}$, Donnacha Kirk ${ }^{3}$, Bob Mann ${ }^{9}$, Jason McEwen ${ }^{10}$, John McKean ${ }^{11}$, Jeffrey \\ Newman $^{12}$, Alvise Raccanelli ${ }^{13,14,15}$, Martin Sahlén ${ }^{6}$, Mario Santos ${ }^{8}$, Anthony \\ Tyson ${ }^{16}$, Gong-Bo Zhao ${ }^{17,1}$ \\ ${ }^{1}$ University of Portsmouth, UK; ${ }^{2}$ University of Manchester, UK; ${ }^{3}$ University College London, \\ UK; ${ }^{4}$ Rhodes University, South Africa; ${ }^{5}$ University of Oslo, Norway; ${ }^{6}$ University of Oxford, UK; \\ ${ }^{7}$ University of Washington, USA $;{ }^{8}$ University of the Western Cape, South Africa $;{ }^{9}$ University of \\ Edinburgh, UK; ${ }^{10}$ Mullard Space Science Laboratory, University College London, UK; \\ ${ }^{11}$ ASTRON, The Netherlands; ${ }^{12}$ University of Pittsburgh, USA; ${ }^{13}$ Johns Hopkins University, \\ USA; ${ }^{14}$ Jet Propulsion Laboratory, Caltech, USA; ${ }^{15}$ California Institute of Technology, USA; \\ ${ }^{16}$ University of California, Davis, USA ${ }^{17}$ National Astronomy Observatories, Beijing, China. \\ E-mail: david.bacon at port.ac.uk
}

\begin{abstract}
We provide an overview of the science benefits of combining information from the Square Kilometre Array (SKA) and the Large Synoptic Survey Telescope (LSST). We first summarise the capabilities and timeline of the LSST and overview its science goals. We then discuss the science questions in common between the two projects, and how they can be best addressed by combining the data from both telescopes. We describe how weak gravitational lensing and galaxy clustering studies with LSST and SKA can provide improved constraints on the causes of the cosmological acceleration. We summarise the benefits to galaxy evolution studies of combining deep optical multi-band imaging with radio observations. Finally, we discuss the excellent match between one of the most unique features of the LSST, its temporal cadence in the optical waveband, and the time resolution of the SKA.
\end{abstract}

Advancing Astrophysics with the Square Kilometre Array

June 8-13, 2014

Giardini Naxos, Italy

\footnotetext{
* Speaker.
} 


\section{The Large Synoptic Survey Telescope}

\subsection{The LSST system}

The LSST system is designed to achieve multiple goals in four main science themes: (i) taking an inventory of the Solar System; (ii) mapping the Milky Way; (iii) exploring the transient optical sky; and (iv) probing dark energy and dark matter. These are just four of the many areas in which LSST will have enormous impact, but they span the space of technical challenges in the design of the system and survey, and have been used to focus the science requirements. The LSST will be a large, wide-field ground-based telescope, camera and data management system designed to obtain multi-band images over a substantial fraction of the sky every few nights. The observatory site will be located on Cerro Pachón in northern Chile (near the Gemini South and SOAR telescopes), with the first light expected in 2021 and the first public data releases mid-2023. The survey will yield contiguous overlapping imaging of over half the sky in six optical bands (ugrizy, covering the wavelength range $320-1050 \mathrm{~nm}$ ).

The LSST telescope uses a novel three-mirror design (modified Paul-Baker) with a very fast f/1.234 beam (Figure 1). The optical design has been optimised to yield a large field of view (9.6 $\mathrm{deg}^{2}$ ), with seeing-limited image quality, across a wide wavelength band. Incident light is collected by the primary mirror, which is an annulus with an outer diameter of $8.4 \mathrm{~m}$ and inner diameter of $5.0 \mathrm{~m}$ (an effective diameter of $6.5 \mathrm{~m}$ ), then reflected to a $3.4 \mathrm{~m}$ convex secondary, onto a $5 \mathrm{~m}$ concave tertiary, and finally into three refractive lenses in a camera. This is achieved with an innovative approach that positions the tertiary mirror inside the primary mirror annulus ring, making it possible to fabricate the mirror pair from a single monolithic blank using borosilicate technology. The secondary is a thin meniscus mirror, fabricated from an ultra-low expansion material. All three mirrors will be actively supported to control wavefront distortions introduced by gravity and environmental stresses on the telescope. The telescope sits on a concrete pier within a carousel dome that is $30 \mathrm{~m}$ in diameter. The dome has been designed to reduce dome seeing (local air turbulence that can distort images) and to maintain a uniform thermal environment over the course of the night.

The LSST camera (Figure 1) provides a 3.2 Gigapixel flat focal plane array, tiled by 189 $4 \mathrm{~K} \times 4 \mathrm{~K}$ CCD science sensors with $10 \mu \mathrm{m}$ pixels. This pixel count is a direct consequence of sampling the $9.6 \mathrm{deg}^{2}$ field-of-view $\left(0.64 \mathrm{~m}\right.$ diameter) with $0.2 \times 0.2 \operatorname{arcsec}^{2}$ pixels (Nyquist sampling in the best expected seeing of $\sim 0.4$ arcsec). The sensors are deep depleted high resistivity silicon back-illuminated devices with a highly segmented architecture that enables the entire array to be read in 2 seconds. The detectors are grouped into $3 \times 3$ rafts; each contains its own dedicated front-end and back-end electronics boards. The rafts are mounted on a silicon carbide grid inside a vacuum cryostat, with an intricate thermal control system that maintains the CCDs at an operating temperature of $180 \mathrm{~K}$. The entrance window to the cryostat is the third of the three refractive lenses in the camera. The other two lenses are mounted in an optics structure at the front of the camera body, which also contains a mechanical shutter, and a carousel assembly that holds five large optical filters. The sixth optical filter can replace any of the five via a procedure accomplished during daylight hours.

The rapid cadence of the LSST observing program will produce an enormous volume of data ( $\sim 15$ TB of raw imaging data per night), leading to a total database over the ten years of operations of $50 \mathrm{~PB}$ for the raw uncompressed imaging data (100PB with processed versions), and 15PB for 

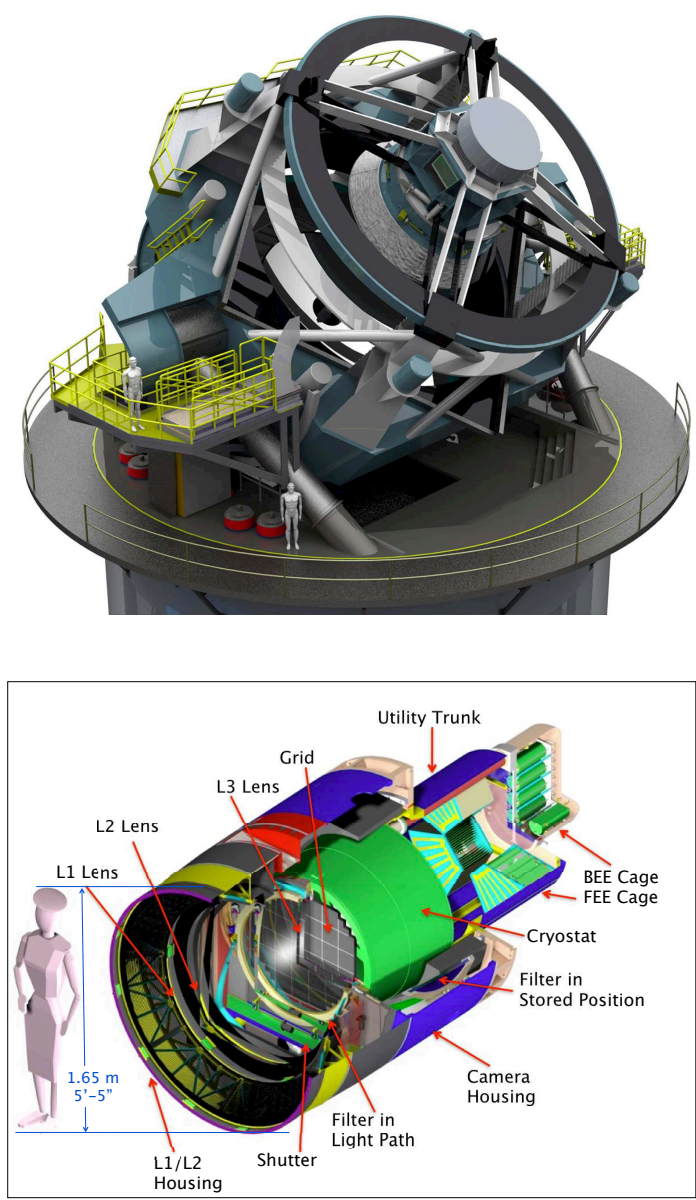

Figure 1: Upper panel: baseline design for the LSST telescope. Lower panel: the LSST camera layout. Credit: LSST Corporation.

the final catalog database. The computing power required to process the data grows as the survey progresses, starting at $\sim 100$ TFlops and increasing to $\sim 400$ TFlops by the end of the survey. Processing such a large volume of data, automated data quality assessment, and archiving the results in useful form for a broad community of users are major challenges. The data management system is configured in three levels: an infrastructure layer consisting of the computing, storage, and networking hardware and system software; a middleware layer, which handles distributed processing, data access, user interface and system operations services; and an applications layer, which includes the data pipelines and products and the science data archives.

The application layer is organised around the data products being produced. The nightly pipelines are based on image subtraction, and are designed to rapidly detect interesting transient events in the image stream and send out alerts to the community within 60 seconds of completing the image readout. The data release pipelines, in contrast, are intended to produce the most completely analysed data products of the survey, in particular those that measure very faint objects and cover long time scales. A new run will begin each year, processing the entire survey data set that is available to date. The data release pipelines consume most of the computing power of the data management system. The calibration products pipeline produces the wide variety of calibration data required by the other pipelines. All of these pipelines are designed to make efficient use of 
computer clusters with thousands of nodes. There will be computing facilities at the base facility in La Serena, at a central archive facility, and at multiple data access centres. The data will be transported over existing high-speed optical fibre links from South America to the USA.

For a more detailed discussion, including optical design, the filter complement, the focal plane layout, and special science programs, please see the LSST overview paper (Ivezic et al. 2008) and the LSST Science Book ${ }^{1}$ (Abell et al. 2009).

\subsection{Planned survey strategy and delivered data products}

The LSST observing strategy is designed to maximise scientific throughput by minimising slew and other downtime and by making appropriate choices of the filter bands given the real-time weather conditions. The fundamental basis of the LSST concept is to scan the sky deep, wide, and fast, and to obtain a dataset that simultaneously satisfies the majority of the science goals. This concept, the so-called "universal cadence", will yield the main deep-wide-fast survey and use about $90 \%$ of the observing time. The observing strategy for the main survey will be optimised for homogeneity of depth and number of visits. In times of good seeing and at low airmass, preference will be given to $r$-band and $i$-band observations. As often as possible, each field will be observed twice, with visits separated by 15-60 minutes. The ranking criteria also ensure that the visits to each field are widely distributed in position angle on the sky and rotation angle of the camera in order to minimise systematic effects in galaxy shape determination.

The current baseline design will allow about $10,000 \mathrm{deg}^{2}$ of sky to be covered using pairs of 15 -second exposures in two photometric bands every three nights on average, with typical $5 \sigma$ depth for point sources of $r \sim 24.5$. For example, these individual visits will be about 2 mag deeper than the SDSS data. The system will yield high image quality as well as excellent astrometric and photometric accuracy for a ground-based optical survey. The survey area will include $30,000 \mathrm{deg}^{2}$ with $\delta<+34.5^{\circ}$, with the $18,000 \mathrm{deg}^{2}$ main survey footprint visited over 800 times during 10 years. The coadded data within the main survey footprint will be 5 mag deeper than SDSS $(r \sim$ 27.5). The main survey will result in databases including 20 billion galaxies and a similar number of stars, which will serve the majority of science programs. The remaining $10 \%$ of observing time will be used to obtain improved coverage of parameter space such as very deep $(r \sim 26)$ observations (e.g. optimised for $\mathrm{SNe}$ ), observations with very short revisit times ( $\sim 1$ minute), and observations of "special" regions such as the Ecliptic, Galactic plane, and the Large and Small Magellanic Clouds.

The LSST data system is being designed to enable as wide a range of science as possible. Standard data products, including calibrated images and catalogs of detected objects and their attributes, will be provided both for individual exposures and the deep incremental data coaddition. About 20 billion objects will be routinely monitored for photometric and astrometric changes, and any transient events (non-recurrent objects with statistically significant photometric change; about 10,000 per night on average) will be distributed in less than 60 seconds via web portals. For the "static" sky, there will be yearly database releases listing many attributes for billions of objects and will include other metadata (parameter error estimates, system data, seeing summary etc).

\footnotetext{
${ }^{1}$ Available from www.lsst.org/lsst/SciBook
} 
LSST has been conceived as a public facility: the database that it will produce, and the associated object catalogs that are generated from that database, will be made available to the U.S. and Chilean scientific communities and public with no proprietary period. Negotiations are under way with prospective international partners to make LSST data more broadly available.

It is expected that the scientific community will produce a rich harvest of discoveries with LSST data products. Many of the highest priority LSST science investigations will require organised teams of professionals working together to optimise science analyses and to assess the importance of systematic uncertainties on the derived results. To meet this need, eleven science collaborations have been established by the project in core science areas. As of the time of this contribution, there are over 500 participants in these collaborations, mostly from LSST member institutions. Through the science collaborations, the astronomical and physics communities are involved in the scientific planning of LSST deployment strategies.

\section{Overview of Synergies}

The SKA and LSST are the two major ground-based survey telescopes of the next decade. They offer significant synergies, in terms of both sky area and time-domain astrophysics, and are likely to be on the sky over much of the same time-period. The survey strategies for SKA1 and SKA2 are being developed in this book, but are likely to include $3 \pi$ surveys over the same area as LSST, as well as thousands of square degrees to a greater depth than LSST.

The LSST will leverage a large range of science from the SKA by providing approximate (photometric) redshifts for about 40 galaxies per square arcminute. Many of the existing SKA science applications assume that such photometric redshift information will be available. LSST is the only foreseen survey covering a large fraction of the southern hemisphere at an appropriate depth to provide this information. Euclid can further improve the photometric redshifts from LSST by supplying infra-red information.

Conversely, the SKA can enable LSST to carry out additional exciting science. For instance, one of the challenges for LSST is to calibrate its photometric redshifts with precise redshift information. The SKA has the potential to provide a large amount of redshift information through observations of HI emission, which can then be used to calibrate LSST objects through crosscorrelation (Newman 2008; McQuinn \& White 2013). SKA will also provide direct redshifts for training photo-zs for highly extinguished or featureless sources that fail to yield emission lines or spectral breaks in the optical/near-IR.

The synergy between the LSST and SKA will have a major impact for several disciplines. In cosmology, much effort is focussed on understanding the apparent accelerated expansion of the Universe. Forecasts currently often take the form of constraints on the dark energy equation of state; however in a decade we may be more concerned with testing the laws of gravity, or investigating new surprises yet to come. In any case, it will be necessary to confront theory with observations of the statistics of cosmological probes, to a large distance. The SKA and LSST are well-suited to each other to obtain the necessary observational cosmology data.

In addition, cosmology in the next decade is likely to have reached the limit of what can be achieved simply by increasing the volume surveyed. Control of systematic errors will be paramount, and therefore cross-checks of quantities between the SKA and LSST will enable ad- 
ditional science. For best results, the data from both surveys can be cross-correlated to reduce systematic effects specific to one survey or the other.

When it comes to our attempts to understand how galaxies form, again we find that this quest is intimately tied to both optical and radio observations. The pivotal role of AGN in galaxy formation (see McAlpine et al. (2015) and Smolčić et al. (2015) in this book), and the presence of neutral hydrogen, can only be understood at radio wavelengths, whereas the star formation history can be extracted from both multi-wavelength optical studies and in an extinction-free way using radio continuum observations (see Jarvis et al. (2015) in this book). LSST provides the necessary photometric redshift information that can be used to simultaneously extract approximate redshifts and star-formation rates.

The SKA and LSST both have unusually high time resolution for survey instruments; both are likely to be able to process data in a matter of seconds. They also have a similar size of instantaneous field of view or beam. Therefore the potential for coordinated surveys to find transients opens up an exciting new regime in observational parameter space.

The SKA and LSST will both benefit greatly from other major facilities that will be observing the southern sky. Facilities such as Euclid and WFIRST will provide key near-infrared wavelength data coupled with high resolution that will aid both strong and weak lensing studies, photometric redshift determination, and will allow estimates of the stellar mass of galaxies at $z>1$. The addition of eROSITA will provide a complementary approach to disentangling the AGN in radio continuum surveys. Complementary to the cosmological studies described here, the proposed all-sky ACTPol survey and future SPT surveys will provide high-resolution CMB polarisation and lensing maps along with a large increase in the number of SZ selected clusters. There are many powerful threeway synergies; here we will concentrate on the SKA-LSST axis.

\subsection{LSST-SKA Methodological Synergies}

Although we focus predominantly on scientific synergies, the LSST and the SKA will also present many methodological synergies. Both experiments will provide petabyte-scale observational datasets recorded over time. Extracting all of the astrophysical information contained in such big data-sets will be a considerable challenge. Although the raw observational data recorded by optical and radio interferometric telescopes exhibit quite different properties, the underlying techniques that will be used to analyse these data share many similarities. Bayesian analysis techniques are now of widespread use in astrophysics (e.g. Lewis \& Bridle 2002; Feroz et al. 2009); sampling methods that scale to very high-dimensional settings, such as Gibbs and Hamiltonian sampling (e.g. Wandelt et al. 2004; Taylor et al. 2008), will be increasingly important in analysing big-data. Machine learning techniques (e.g. Ball \& Brunner 2010) will also play an increasingly important role in tackling the curse of dimensionality that both LSST and SKA data will suffer. Supervised machine learning techniques can be exploited to efficiently navigate these high-dimensional datasets, while unsupervised learning techniques can be used for dimensionality reduction, allowing the data to effectively speak for themselves.

The sparse structure of big data-sets can also be exploited. Compressive sensing (Candès 2006; Donoho 2006) is a recent ground-breaking development in information theory, going beyond the Shannon-Nyquist sampling theorem by exploiting sparsity, and which has the potential to revolutionise data acquisition in many fields (for a brief introduction see Baraniuk 2007). Although the 
application of compressive sensing techniques in astrophysics is not yet mature, first applications for radio interferometric imaging have shown considerable promise (e.g. Carrillo et al. 2014). The effective application of all of these analysis methodologies will be instrumental in the extraction of scientific information from large observational data-sets, such as those recorded by LSST and the SKA. Both experiments will benefit greatly from sharing expertise, analysis techniques and open-source numerical codes.

\section{Cosmology with LSST and SKA}

Here we will discuss the synergies we expect for major cosmological probes, in particular galaxy clustering (including intensity mapping), weak lensing and strong lensing.

\subsection{Weak lensing}

LSST will have an unprecedented sample of 3 billion galaxies with high $\mathrm{S} / \mathrm{N}$ and good colourredshifts with which to generate dark matter tomographic maps. SKA1 can obtain 40 million galaxies for weak lensing, with SKA2 possibly matching LSST's number density (see Brown \& et al. 2015, in this book). Overlapping optical and radio surveys such as those carried out by LSST, SKA1-MID and SKA2 have a particularly useful synergy in terms of reducing and quantifying the impact of systematic effects in weak gravitational lensing analyses (Brown \& et al. 2015). By cross-correlating the shapes of galaxies as measured in the optical and radio surveys, one can eliminate instrumental systematic effects that are not correlated between the two telescopes (Patel et al. 2010). Given the very different designs and modes of operation of optical and radio telescopes, one would not expect their instrumental systematic effects to be correlated, and so this offers a route to measuring the cosmic shear signal in a very robust way.

In addition, radio surveys offer unique ways to measure the lensing signal that are not available to optical telescopes. In particular, both radio polarisation information and rotational velocity measurements from HI observations can provide estimates of the intrinsic position angles of the lensing source galaxies (Blain 2002; Morales 2006; Brown \& Battye 2011). Such measurements offer great potential to (i) reduce the effects of galaxy "shape noise" (due to the intrinsic dispersion in galaxy shapes) and (ii) to mitigate the contaminating signal from the intrinsic alignments in galaxy orientations which is perhaps the most worrisome astrophysical systematic effect facing future weak lensing surveys. In addition to using this information in a combined analysis, one could potentially use the SKA-based estimates of the intrinsic alignment contamination to calibrate out the alignment signal in the LSST lensing survey.

Finally, the envisaged SKA1-MID and SKA2 surveys will probe a wider range of redshifts than will be reached by LSST. They therefore provide extra (high-redshift) tomographic slices with which the evolution of structure at relatively early times can be probed. SKA can also push to high redshift by measuring the lensing distortion signal in HI intensity mapping surveys (Pourtsidou \& Metcalf 2014). Thus, these high-redshift SKA lensing experiments will naturally fill the gap between the traditional optical lensing probes (where sources are typically located at $z \sim 1$ ) and the ultimate lensing source of the CMB at $z \sim 1000$. 


\subsection{Galaxy clustering}

The three approaches to SKA galaxy clustering benefit from LSST in distinct ways, which we will now describe.

\subsubsection{HI galaxy survey}

As discussed in the chapter on HI threshold surveys (Abdalla et al. 2015), one approach to galaxy clustering with SKA is to measure redshifts from the HI line for a sample of individually detected galaxies. This provides us with a galaxy survey where the redshifts are known to very high precision (we assume a Gaussian error of $\delta_{z}=0.0001$ ). There is a powerful synergy here between this spectroscopic-quality large scale structure (LSS) survey from SKA and the weak gravitational lensing (WGL) surveys from LSST (with or without the further improvements in systematics from combining with the SKA weak lensing survey, see section 3.1 above). The LSST WGL survey has poor redshift resolution (due both to photometric-quality redshifts and the inherently broad WGL geometric kernel) but has direct access to the true matter distribution in the Universe. In contrast the SKA LSS survey has very good redshift resolution but uses galaxies as biased tracers of the mass distribution. Combination of the two surveys allows us to control for uncertainties in galaxy bias and improve our knowledge of how mass clustering evolves with redshift compared to WGL alone.

In Fig. 2 we present results combining a specimen SKA LSS survey with an LSST WGL survey. Our WGL survey is assumed to have a source density of $n_{g}=50 \mathrm{arcmin}^{-2}$ and to cover a redshift range of $0<z<3.0$. The galaxy redshift distribution is given by Hojjati et al. (2012) and broken into 6 tomographic bins of approximately equal number density. The Gaussian scatter on the photo-z errors is $\delta_{z}=0.03$.

We assume a LSS study with the full SKA2 survey over 30,000 $\mathrm{deg}^{2}$. We have adopted the galaxy redshift distribution discussed in Santos et al. (2014); our forecasts assume 40 tomographic bins up to $z=2.0$. We use the exact projected angular power spectrum, $C(\ell)$, formalism (not the Limber approximation) and include the effects of redshift space distortions (RSDs) according to the formalism of Kaiser (1987). Both these effects are neglected in the WL forecasts because the broad tomographic bins make their impact negligible. We use a maximum wavenumber of $\ell_{\max }=3000$ for the WL analysis, and exclude non-linear scales in the LSS analysis using the cutoff $\ell_{\text {max }}\left(z_{\text {medi }}\right)=k_{\text {lin max }}\left(z_{\text {medi }}\right) \chi\left(z_{\text {med i }}\right)$. Here $z_{\text {med i }}$ is the median redshift of tomographic bin $i, k_{\text {lin max }}\left(z_{\text {med }}\right)=0.132 z_{\text {med }} h^{-1} \mathrm{Mpc}$, and $\chi$ represents comoving distance.

Our Figure of Merit analysis forecasts constraints for a set of cosmological parameters: $\left\{\Omega_{m}\right.$, $\left.\Omega_{b}, \Omega_{D E}, w_{0}, w_{a}, h, \sigma_{8}, n_{s}, b, Q_{0}, Q_{0}\left(1+R_{0}\right) / 2\right\}$. As well as the standard wCDM parameters, $b$ is a free amplitude on galaxy bias and $Q_{0}, Q_{0}\left(1+R_{0}\right) / 2$ are parameterisations of deviations to General Relativity that modify the Poisson equation and the ratio of metric potentials; our ability to constrain these parameters quantifies our ability to test gravity on cosmic scales, see e.g. Kirk et al. (2013). When presenting constraints on dark energy we marginalise over the cosmological parameters and galaxy bias but keep the modified gravity parameters fixed. When presenting constraints on modified gravity we marginalise over cosmology, including $w_{0}$ and $w_{a}$, and galaxy bias. Priors consistent with the latest Planck temperature constraints are included. 

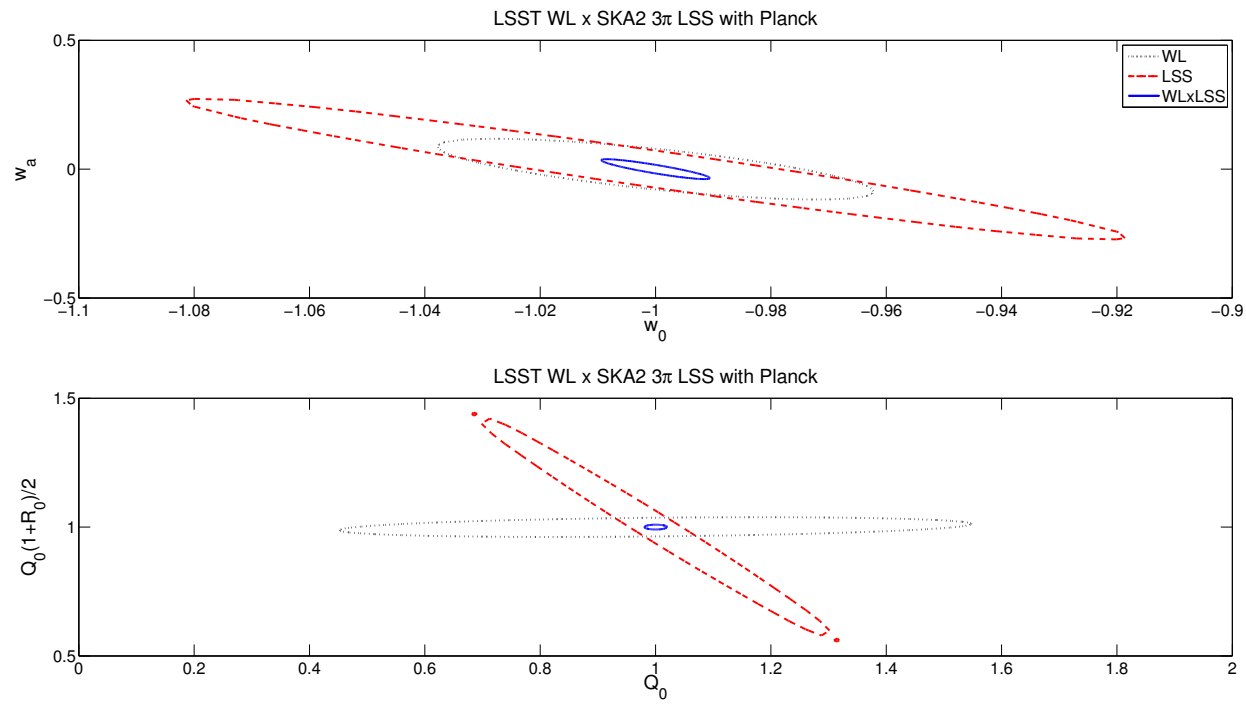

Figure 2: Constraints on dark energy (top panel) and gravity parameters (bottom panel) with SKA2 HI galaxy survey and LSST WGL survey, together with Planck priors.

It is clear from Fig. 2 that the combination of SKA2 and LSST yields an impressive constraining power. These probes are very complementary; the WGL survey responds directly to the presence of matter, but has poor discrimination in redshift due both to the reliance on photometric redshift and the irreducible width of the lensing kernel along the line of sight. In contrast the LSS survey uses galaxies as biased tracers of the underlying dark matter distribution but has much greater resolution in redshift. The combined analysis makes use of the best features of both probes, gaining redshift discrimination from LSS while directly probing the growth of structure and the geometry of expansion through WGL.

Of course it is possible that systematic effects might affect each probe differently and lead to mis-aligned probability ellipses in the upper panel of Fig. 2, so careful tests and cross-checks for systematics are essential. On the other hand, another benefit of combined probe analysis is the ability to "calibrate" systematics of one probe using another. For example, galaxy Intrinsic Alignments (IAs) could be an important astrophysical contaminant of WGL measurements; but information from spectroscopic LSS surveys can be used to down-weight physically close galaxy pairs and mitigate the impact of IAs.

The improvement over each probe alone is particularly pronounced in the modified gravity constraints; this is the result of combining one probe sensitive to the bending of light (WL, sensitive to the sum of metric potentials $\Psi+\Phi$ ) and another probe using galaxies as non-relativistic tracers (LSS, sensitive to the Newtonian potential $\Psi$ ). This combination of sensitivities breaks a pronounced degeneracy in the MG parameter space and produces constraints far stronger than either probe alone.

\subsubsection{Principal Component Analysis}

The dark energy and gravity parameters constrained above are constant physical quantities as a 
function of time and scale. Instead, we can conduct the Principal Component Analysis (PCA) approach from the chapter by Zhao et al. (2015) to examine how sensitive the LSST and SKA are to physical quantities. In particular, we can examine the sensitivity to the time evolution of the dark energy equation of state, $w(z)$, and the time and scale dependence of the effective Newton's constant $\mu(k, z)$, and the gravitational slip, $\gamma(k, z)$.

The details of our approach are given in Zhao et al. (2015). We consider several LSST cosmological probes: weak gravitational lensing, clustering, and the clustering-lensing cross-correlation; we use the number density and bias models given in Hojjati et al. (2012). In addition, we use the HI clustering surveys for SKA1-MID and SKA2, with number density, bias and survey parameters as in Santos et al. (2014), Tables 2 and 4). We make PCA analyses for each telescope independently in combination with Planck constraints, or in combination with each other and with Planck.

Firstly, we work in the context of General Relativity $(\mu=1, \gamma=1)$ and calculate the Fisher matrix for each telescope combination, with the cosmological parameters $\left\{\Omega_{b} h^{2}, \Omega_{c} h^{2}, h, \tau, n_{s}, A_{s}, w_{i}\right\}$. After marginalising over the other parameters, we perform PCA on the $w_{i}$ bins. The results are shown in Figure 3; we see that LSST+Planck is already excellent at constraining eigenmodes of the dark energy equation of state, with 5 modes constrained at the $\sigma<0.1$ level. SKA1 and SKA2 alone are not competitive for this purpose; however, SKA1 in combination with LSST substantially improves LSST constraints on the first few modes (by 70\% for SKA1 and 75\% for SKA2, for the best constrained mode).

Next, we allow $\mu$ and $\gamma$ to vary as a function of time and scale, in addition to dark energy time variation. The results of the various PCA analyses, described in detail in Zhao et al. (2015), are shown in Figure 4; in this case, the synergy between LSST and SKA is very strong. While LSST+Planck alone can constrain 5 modes of the effective Newton constant $\mu$ at the $\sigma<0.01$ level, even LSST+SKA1+Planck can constrain 7, and LSST+SKA2+Planck can constrain 11 with errors on the first mode reduced by a factor of 5 . The situation is equally impressive for the gravitational slip $\gamma$; the addition of SKA1 to LSST+Planck reduces errors on the first mode by $20 \%$, and the addition of SKA2 reduces errors by a factor of 6 .

As in Section 3.2.1, the cause of this improvement is the different physical effects of $\mu$ and $\gamma$ on different cosmological probes. Weak lensing and the CMB are sensitive to combinations of the two metric potentials $\Phi$ and $\Psi$, which are affected by both $\mu$ and $\gamma$ modes. On the other hand, redshift space distortions measured in the SKA HI surveys are only sensitive to $\Psi$, and hence $\mu$. Thus we find that LSST+SKA are an excellent combination for future tests of gravity.

\subsubsection{Continuum survey}

As described in the chapter on cosmology with radio continuum surveys (Jarvis et al. 2015), a new era for continuum cosmology is close to becoming a reality. Among the numerous planned surveys are the LOFAR Surveys, the Evolutionary Map of the Universe (EMU), the MeerKAT-MIGHTEE survey and the Westerbork Observations of the Deep APERTIF Northern Sky (WODAN). These forthcoming experiments will provide us with a homogeneous all-sky continuum catalogue $>10$ times larger than the largest one hitherto available, and SKA1 will be able to reach a factor an order of magnitude deeper over similar sky areas to these.

However, radio continuum surveys do not provide any redshift information for the sources. For cosmological purposes, this is a serious issue; to investigate cosmic acceleration, we require 


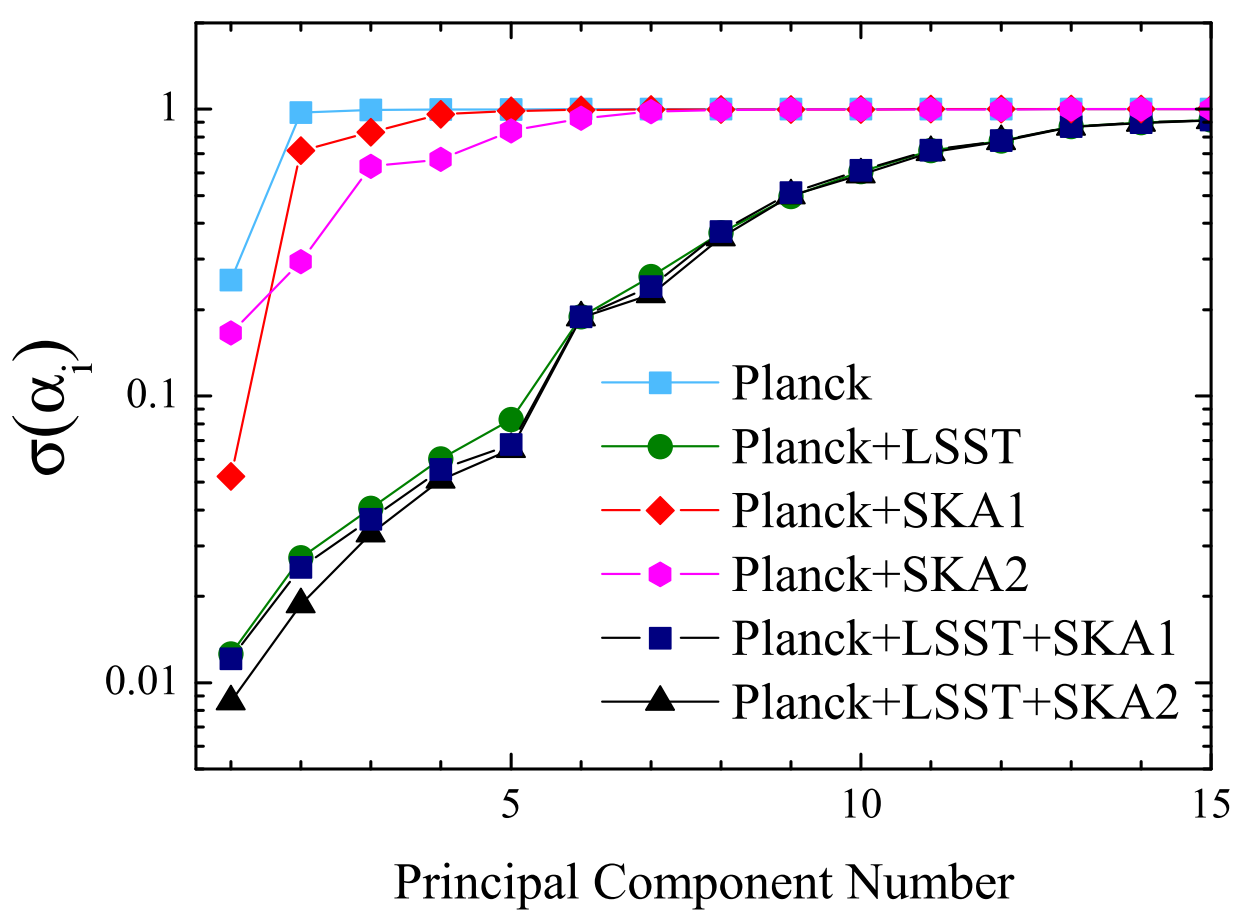

Figure 3: The forecast 68\% CL measurement error of the principal components, for the dark energy equation of state, for different survey combinations listed in the legend.

information about the time evolution of the Universe's expansion and structure growth. For this purpose, Camera et al. (2012) proposed to cross-identify continuum radio sources with optical to near-infrared surveys (currently these include SkyMapper and SDSS). Even now such studies could be extended by incorporating data from other surveys, particularly at near/mid-infrared wavelengths where the VISTA Hemisphere Survey (McMahon et al. 2013), 2MASS (Skrutskie et al. 2006) and WISE (Wright et al. 2010) can provide robust detections of low-redshift sources. LSST will further improve the prospects for cross-identification at higher redshift. By making these cross-matches, one can separate the source distribution into a low- and a high-redshift sample, thus providing information on the evolution of cosmological parameters such as those related to dark energy. This approach yields constraints more than four times tighter than in the case without redshift information.

Using both the SKA and the LSST, we are also able to perform a cross-correlation of galaxy clustering with the Cosmic Microwave Background, probing the integrated Sachs-Wolfe (ISW) effect. Raccanelli et al. (2014) have provided promising forecasts for constraining the non-Gaussianity of primordial fluctuations using this probe with SKA and photometric surveys. This can be seen from Figure 5, where we show how having redshift information will enable a tomographic ISW, which will help in pinning down the constraints on the non-Gaussianity parameter $f_{\mathrm{NL}}$. We plot constraints for the SKA1 only case (no redshift information) for a $5 \mu \mathrm{Jy}$ rms survey of $30000 \mathrm{sq}$ deg, and where redshift information is provided by a photometric survey such as LSST for SKA 


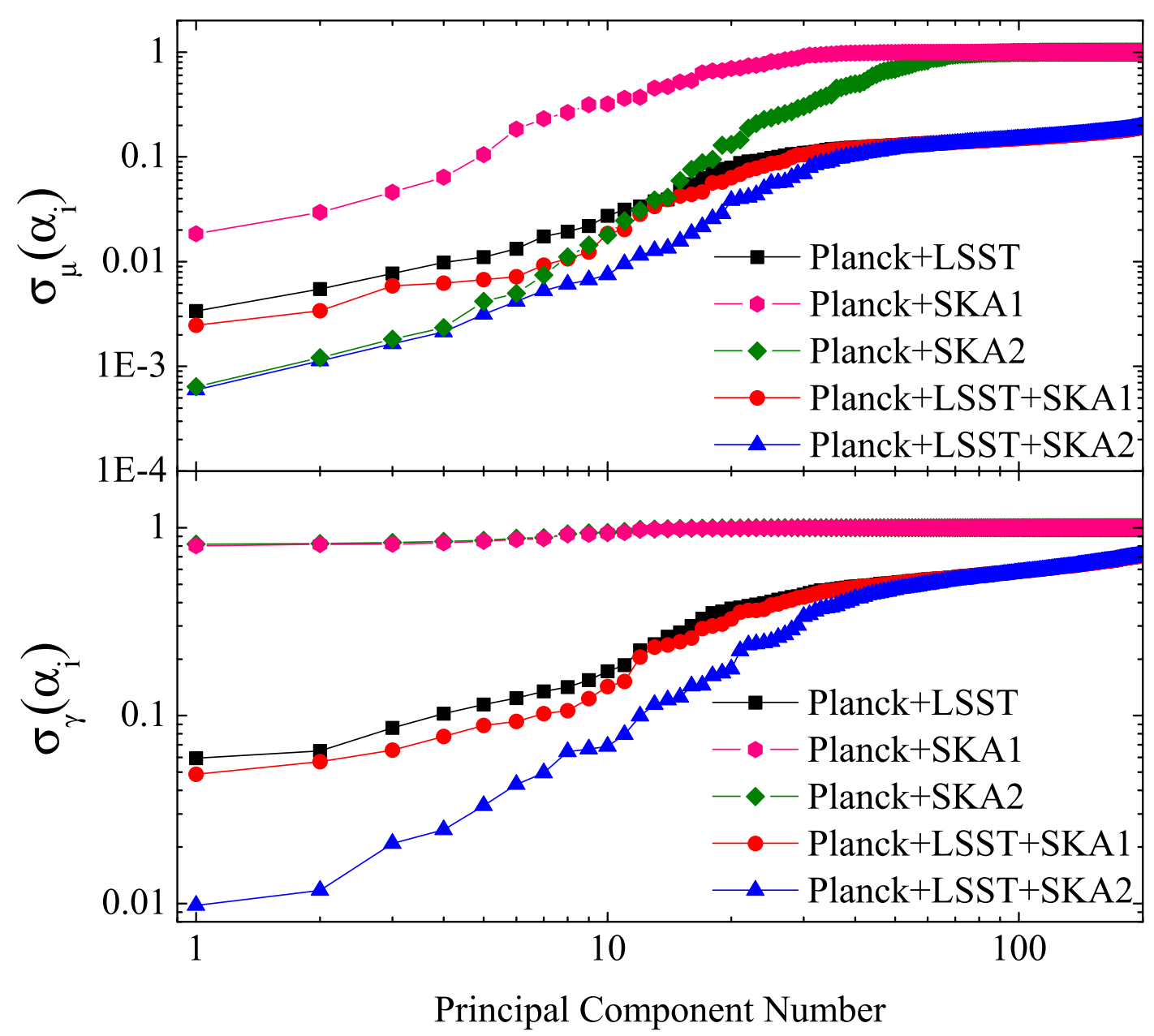

Figure 4: The forecast $68 \% \mathrm{CL}$ error of the principal components of $\mu(k, z)$ (top panel) and $\gamma(k, z)$ (bottom panel), for different survey combinations listed in the legend.

sources, up to $z=1$ and $z=2$. The error on $f_{\mathrm{NL}}$ is reduced from 20 to $\simeq 1$, which compares favourably to the current current best constraint of $f_{\mathrm{NL}}=2.7 \pm 5.8(1 \sigma)$ from Planck (Ade et al. 2014).

\subsubsection{Intensity mapping}

Intensity mapping of the redshifted neutral hydrogen $21 \mathrm{~cm}$ emission line is an exciting new survey methodology for large scale structure. Instead of resolving many individual galaxies at high signalto-noise, one instead uses low-resolution maps of the integrated emission from many unresolved 


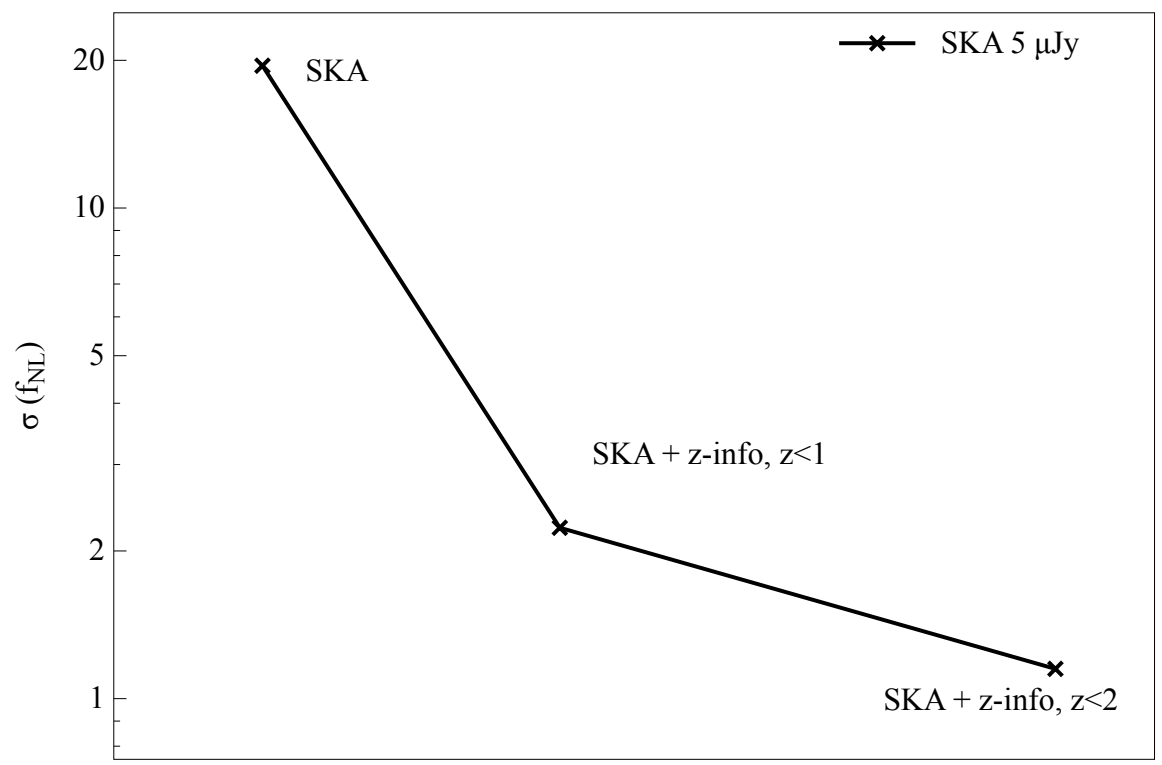

Figure 5: Constraints on the non-Gaussianity parameter $f_{\mathrm{NL}}$ from the cross-correlation SKA-CMB, with and without redshift information provided by a photometric survey such as LSST.

galaxies to probe the large scales $\left(\sim 1^{\circ}\right)$ corresponding to the baryon acoustic oscillations. Since the galaxy population hosting the $\mathrm{HI}$ emitting regions is a biased tracer of the underlying dark matter distribution, so too is the integrated HI signal, and redshift information is trivially obtained by the frequency of the emission. By making low-resolution maps over a substantial fraction of the sky, for many channels over a wide band, intensity mapping (IM) surveys are therefore able to rapidly reconstruct the large-scale redshift-space matter distribution over extremely large cosmological volumes, out to high redshift (Santos et al. 2015).

While the IM methodology is not yet mature, a number of medium-size experiments that are either planned or in construction (e.g. CHIME, Bandura et al. (2014), BINGO, Battye et al. (2012)) are expected to yield cosmological results substantially before SKA1 sees first light. With SKA1, a 10,000 hour, 25,000 deg ${ }^{2}$ IM survey on either the MID or SUR arrays are projected to yield dark energy constraints that are competitive with a Dark Energy Task Force Stage IV galaxy redshift survey (Bull et al. 2014; Bull \& et al. 2015; Raccanelli et al. 2015). This could potentially be completed several years before LSST (and even Euclid), with a substantial overlap in survey area and redshift coverage.

While this would provide many of the same advantages as an overlapping galaxy survey in the radio, intensity mapping has a number of additional benefits in terms of synergies with LSST. Foremost is the significantly different set of systematic effects that one expects from making intensity maps rather than galaxy catalogues. A number of calibration and selection effects that are common to galaxy surveys, but not IM, can then be expected to drop out in cross-correlation, which will be especially powerful if, as expected, future large scale structure surveys are systematics-limited. IM surveys are also capable of spanning both lower and higher redshifts than LSST, and can potentially be used to 'anchor' the LSST data by filling in some information in missing redshift bins - 
for example, SKA1-MID can cover $0.4 \lesssim z \lesssim 3$ with Band 1 , and $0 \lesssim z \lesssim 0.5$ with Band 2 .

Because IM surveys probe a differently-biased population of galaxies to LSST, one can also benefit from multi-tracer analysis (McDonald \& Seljak 2009; Abramo \& Leonard 2013; Camera \& et al. 2015). For certain observables - most notably redshift space distortions - this allows the limits imposed by cosmic variance to be beaten, which is of particular importance for reconstructing the growth history of the Universe to high enough precision to test modifications to General Relativity (Raccanelli et al. 2015).

\subsection{Strong lensing}

The 2020s will be a new era for studying galaxy formation, the high redshift Universe and cosmology with strong gravitational lensing (see the chapter by McKean \& et al. (2015) in this volume). Currently, $\sim 500$ strong lens systems are known, of which about $10 \%$ are radio-loud. This sample will potentially increase with SKA and LSST to about 100000 in each waveband (e.g. Marshall et al. 2005; Oguri \& Marshall 2010). SKA alone will detect lensed sources in abundance, with a few per square degree accessible to SKA1 and many more to SKA2. These will include AGN and star forming regions via their continuum synchrotron emission, and gas clouds out to high redshift via their molecular line emission. The latter are particularly interesting for providing source redshifts and velocity fields.

Detailed multi-band optical and infrared imaging will be important both for finding and using this new sample of gravitational lenses. Having two surveys at very different wavebands reduces the false positive rate, which is likely to be the main problem with the next generation of lens surveys, because the radio-optical flux ratio of potential multiple lensed images will be a much better discriminant than anything available within a single waveband. ( 0.3 "-resolution optical data would have drastically reduced the necessary followup in radio surveys such as CLASS (Myers et al. 2003), for instance). A secondary consideration is that detecting the lensing galaxy allows probabilistic arguments to be made about the likelihood of a lensing model for any surrounding objects, versus the hypothesis that the surrounding objects are non-lensed features such as starforming regions. The deep multi-band photometry from the LSST will enable the lensing galaxies to be detected and their photometric redshifts to be measured in most of the SKA systems; lens galaxy redshifts and positions are needed for accurate modelling of their mass distributions. Once we have confirmed lens systems with good lens models, we will be able to test galaxy formation models, and explore source populations at high magnification.

The synergy between the LSST and SKA is also important for the cosmological applications of strong lenses. Both telescopes will be capable of measuring independent gravitational time-delays from the variable optical and radio emitting regions, needed for the measurement of distances and so to test models for dark energy (e.g. Suyu et al. 2010). Multi-wavelength follow-up is very important in these studies, to characterise the properties of the lenses and their environment, which are needed to overcome systematics in the mass model. The radio monitoring is particularly powerful where the source is varying, since the lightcurves are not (or at least much less) affected by microlensing. 


\section{Galaxy evolution with LSST and SKA}

With the onset of wide and deep field surveys across all wavebands, coupled with high-resolution cosmological hydrodynamic simulations, much progress has been made in understanding galaxy evolution over the past decade. However, there are clear deficiencies in our understanding of galaxies over the whole of cosmic time. At the bright end of the galaxy luminosity function, we need to understand better the role of AGN feedback (Fabian 2012), and how this may truncate or stimulate star formation in the AGN host itself or in the surrounding environment. At the faint end, we need to understand the influence of the environment, and how for instance hot and cold gas may get stripped from galaxy haloes as they fall into the deep potential wells of clusters.

The combination of deep optical imaging from LSST, coupled with both continuum and spectral line surveys with the SKA, offers a unique opportunity to significantly impact on our understanding of the formation and evolution of galaxies up to the highest redshifts. Here we highlight some of the key synergies that are also discussed at greater length in other chapters in this volume.

\subsection{The evolution of activity in the Universe}

One of the key goals of the SKA continuum surveys is to provide a complete census of the AGN and star-formation activity across cosmic time. Radio continuum observations play a unique role for both of these topics. For AGN, the radio traces highly energetic jets which provide the mechanical feedback in many semi-analytic models and hydrodynamic simulations of galaxy evolution. However, current radio surveys do not provide a full picture of the impact of such sources. In particular, more depth is required to obtain information on the detailed physics of distant radio sources, coupled with a full sampling of the uv-plane to ensure that both large and small spatial scales are sampled as required. On the other hand, the sources are rare and thus relatively wide surveys are also needed to ensure that all environments at all redshifts are fully sampled.

Radio emission may provide the most robust tracer of the star-formation rate in all galaxies. This is because the radio waveband does not suffer from dust extinction which is a limiting property of similar studies using the visible waveband. Furthermore, the high-resolution, sub-arcsecond imaging possible with the SKA ensures that AGN and star-formation activity can be distinguished, and that radio sources can be associated with optical and near-infrared counterpart galaxies.

Therefore, continuum surveys with the SKA provide the necessary depth and area to provide a complete census of AGN and star-formation activity across cosmic time. However, what is missing from radio observations is information on the redshift, stellar mass, and the many other properties associated with the stellar populations within galaxies (e.g. metallicity, age) which can be supplied by LSST. On the other hand, knowledge of the presence of an AGN from the SKA will ensure that AGN emission can be considered for modelling the SED at optical/near-infrared wavelengths. Thus it is only by combining the SKA continuum surveys with photometric redshifts and stellar properties from the LSST that we will gain the fullest picture of galaxy evolution.

The LSST Deep Drilling Fields will provide extremely deep imaging data ( $\mathrm{AB} \sim 28 \mathrm{mag}$ ) in four extragalactic fields, overlapping with deep near-infrared imaging from UltraVISTA (McCracken et al. 2012) and VIDEO (Jarvis et al. 2013) of LSST ( $9.6 \mathrm{deg}^{2}$ each). The LSST Deep Drilling Fields combined with the proposed deep SKA continuum fields will allow us to trace Milky Way type galaxies up to the highest redshifts with both facilities, sampling enough cosmological 
volume not to be dominated by sample variance issues for this purpose. In particular, these LSST deep fields will be sufficiently deep to find galaxies at $z>6$, which will be ripe for $\mathrm{CO}$ redshift measurement with SKA Band 5. The LSST all-sky survey coupled with the SKA all-sky survey will provide a similar census of galaxy evolution at $z<1$, again sampling all of the accessible volume.

\subsection{The evolution of hydrogen}

One of the unique aspects of the SKA is its ability to measure the evolution of neutral hydrogen in the Universe. With SKA1 this can be done in deep fields out to the limiting frequency for SKAMID (350MHz corresponds to a redshift of $z \sim 3$ ). In the wider area surveys the ability to detect HI will probably be limited to around $z \sim 0.6$.

Together the SKA and LSST surveys could potentially provide the key information on how quickly gas is turned into stars. For example, in combination with ALMA, the surveys could provide a continuous view of the path from neutral (SKA) to molecular gas (ALMA) through to star formation (radio continuum and LSST). To be able to carry out such studies as a function of galaxy mass, galaxy type and environment, will strongly enhance our understanding of the evolution of galaxies.

\subsection{High-redshift galaxies and reionization}

The LSST galaxy sample at $z \sim 5$ will provide an important calibration of stellar mass density and galaxy clustering, as a function of galaxy properties and environment. These can anchor interpretations of measurements of the brightness temperature fluctuations from the epoch of reionization with SKA. During the later stages of reionization, the brightness temperature is dominated by fluctuations in the neutral hydrogen fraction, which in turn depend on reionization-source properties and their clustering statistics (Mellema et al. 2013). Measurements of such quantities during the epoch of reionization, $z=6-15$, with instruments such as VISTA, James Webb Space Telescope, and Hyper-SuprimeCam, will need such a lower-redshift normalization around the end of reionization to realize their full statistical potential. Deep observations with the James Webb Space Telescope to detect samples of the first galaxies will particularly complement the LSST sample by providing an anchoring point at the start of, or before, the epoch of reionization. The combination of the LSST, SKA, and James Webb Space Telescope will be key for joint constraints on galaxy evolution and reionization.

The LSST Deep Drilling Fields will provide additional information during the epoch of reionization, $z>6$, in principle allowing studies of cross-correlation and co-evolution between galaxies and the brightness temperature/neutral hydrogen fraction from SKA. In combination with potential deep SKA continuum fields and complementary near-infrared data, the Deep Drilling Fields will allow the determination of key source population observables such as UV luminosity, star formation rate, escape fraction of ionizing photons, metallicity, and stellar mass (e.g. in combination with Spitzer or Euclid). Additional redshift measurements from SKA would complement this.

As a baseline, by combining the source population data with the measured global brightness temperature signal from SKA, constraints can be placed on the fraction of reionization that is provided by galaxies. If reionization occurs late, at $z \sim 6-10$ as some evidence suggests, e.g. Pentericci (2012), such that the brightness temperature fluctuations still trace the source statistics, 


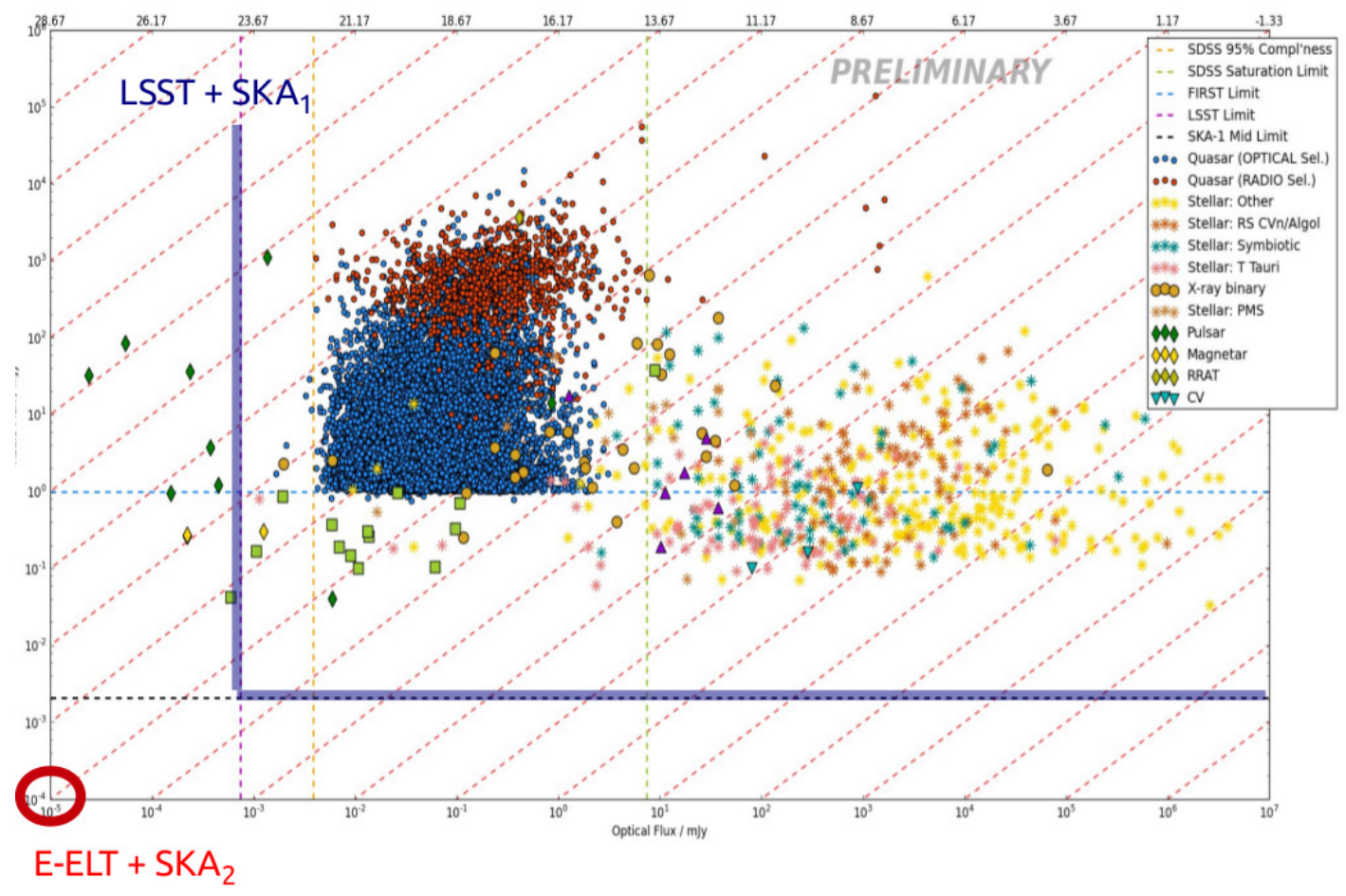

Figure 6: Radio vs optical flux density for a range of astrophysical variables, including a large set of SDSS AGN as well as stellar sources, pulsars and and active binaries. The sample is largely cut off by the limits of the SDSS and FIRST optical and radio surveys, respectively. Optical flux is immediately seen to be a good discriminator between the broad classes of source. The solid mauve lines indicate the typical nightly sensitivities of LSST and SKA1-MID for a given field, demonstrating how much further into parameter space they will routinely push. For example, stellar sources will be detectable throughout the galaxy instead of just locally. Also indicated are the flux limits for a typical joint observation of a field with E-ELT and the dish component of SKA2 (albeit rather uncertain). From Stewart et al., in prep.

cross-correlation of the LSST Deep Drilling Fields with SKA might be possible (Lidz et al. 2009; Wiersma et al. 2013). This would provide another way to constrain details of the reionization process, e.g. to what degree different galaxy types are responsible. Since LSST will characterise the source galaxy populations in great detail, the statistics of the brightness temperature field could also be directly correlated with galaxy properties. Such information is important for a full characterisation of the power spectrum of brightness temperature fluctuations, and hence for extracting the full cosmological information from SKA (Mellema et al. 2013).

\section{Variables and transients}

One of the key science drivers for the LSST is the time variable Universe and transient astrophysics. 
In recent years this is an area which has also come to the fore in the key programmes being planned or implemented on the SKA and its pathfinders and precursors. LOFAR (Stappers et al. 2011), MeerKAT, ASKAP and the MWA all have approved key programmes in the area of radio transients, both 'fast' (coherent, found primarily in beamformed data) and 'slow' (incoherent synchrotron, found mainly in image stacks). The chapters in this book by Fender et al. (2015); Macquart et al. (2015); Corbel \& et al. (2015) provide an overview of the science and likely performance of the SKA transients surveys in both modes, with further, more detailed, case studies in the following transient chapters. An earlier review of the prospects for radio transients with the SKA can be found in Fender \& Bell (2011).

However, it is clear that the value of finding radio transients is severely reduced if counterparts at other wavelengths are not also identified. Since the sources are by definition ephemeral, this usually means rapid identification, reporting and follow-up of radio transients (hence the strong push for automated commensal transient searching in Fender et al. (2015)). Which wavelengths are best? In most cases, although X-ray and other counterparts will be important, the optical or infrared bands are most needed, as these can be readily compared against reference images and can identify candidates for spectroscopic follow-up (and possibly redshift measurement) in the most exciting cases. Probably the single highest priority, therefore, for radio transient follow-up, is to get an optical photometric measurement. Fig. 6 presents a wide sample of the optical and radio fluxes, from sources ranging from stars to supermassive black holes, which are likely radio variables.

Fortunately, in the era of parallel wide-field SKA and LSST observations, the optical data will be readily available for most fields. For the kind of surveys being envisaged with SKA1, and the nightly sky sweeps of LSST, several fields per night will get much deeper than the parameter space explored in Fig. 6 (mauve lines).

\section{Conclusions}

In this chapter we have examined the value of combining data and analyses with the SKA and LSST. We introduced the LSST, recognising it as one of the foremost survey telescopes of the next decade. We then discussed the synergies available between the SKA and LSST, at both the methodological and science result levels.

In the field of cosmology, we discussed how weak gravitational lensing benefits from the combination of optical and radio shape measurements with radically different systematic effects present. We showed how the combination of LSST lensing and clustering, and SKA galaxy clustering with spectroscopic redshifts, provide improved constraints on dark energy and gravity parameters. We discussed how the synergy between LSST and SKA continuum and intensity mapping measurements also provides improvements on cosmological parameters. Strong lensing studies benefit from the combined ability of SKA and LSST to characterise both the lenses and sources in detail.

We have also discussed the benefits to studies of galaxy evolution, where SKA can provide information on redshift, neutral hydrogen, AGN and star formation, while LSST can provide complementary information about star formation, galaxy age and metallicity. LSST will provide an important calibration of galaxy properties for interpreting brightness temperature fluctuation data 
obtained with SKA from the epoch of reionization. The LSST Deep Drilling Fields in combination with SKA will help determine how galaxy populations reionize the Universe, thereby also providing information that will improve the SKA cosmology analysis.

Finally, we have discussed the value of combining the LSST and SKA time domain in order to understand and discover a wide range of astrophysical variables and transients.

In conclusion, using both surveys gives:

- Complementary physical constraints (e.g. the sensitivity of LSST lensing to both metric potentials, and the SKA RSD measurements to the Newtonian potential alone);

- Removal of systematics (e.g. cross-correlation of optical and radio lensing signals removing shape measurement systematics);

- Cross checks of results; in the forthcoming period of concern about systematic effects, even using the two machines independently will provide important verification of the science results (c.f. the experiments at the Large Hadron Collider);

- Mutual science support (e.g. LSST providing photometric redshifts for SKA continuum detections, and SKA calibration of LSST photo-zs via spectroscopic redshift cross-correlations);

- A more complete picture (e.g. in galaxy evolution, LSST and SKA are sensitive to different components of the galaxy such as neutral hydrogen and stellar populations; in the time domain, the telescopes inform us of different aspects of the transient physics.)

\section{References}

Abdalla, F. B., Bull, P., Camera, S., et al., 2015, "Cosmology from HI galaxy surveys with the SKA", in proc. Advancing Astrophysics with the Square Kilometre Array, PoS(AASKA14)017

Abell, P. A., Allison, J., Anderson, S. F., et al. 2009, arXiv:0912.0201

Abramo, L. R., \& Leonard, K. E. 2013, MNRAS, 432, 318

Ade, P. A. R., Aghanim, N., Armitage-Caplan, C., et al. 2014, A\&A, 571, A24

Ball, N. M., \& Brunner, R. J. 2010, International Journal of Modern Physics D, 19, 1049

Bandura, K., Addison, G. E., Amiri, M., et al. 2014, in Society of Photo-Optical Instrumentation Engineers (SPIE) Conference Series, Vol. 9145, Society of Photo-Optical Instrumentation Engineers (SPIE) Conference Series, 22

Baraniuk, R. 2007, IEEE Signal Processing Magazine, 24, 118

Battye, R. A., Brown, M. L., Browne, I. W. A., et al. 2012, arXiv:1209.1041, arXiv:1209.1041

Blain, A. W. 2002, ApJLett, 570, L51

Brown, M., Bacon, D. J., Camera, S., et al., 2015, "Weak gravitational lensing with the Square Kilometre Array", in proc. Advancing Astrophysics with the Square Kilometre Array, PoS(AASKA14)023

Brown, M. L., \& Battye, R. A. 2011, MNRAS, 410, 2057

Bull, P., Camera, S., Raccanelli, A., et al., 2015, "Measuring baryon acoustic oscillations with future SKA surveys", in proc. Advancing Astrophysics with the Square Kilometre Array, PoS(AASKA14)024 
Bull, P., Ferreira, P. G., Patel, P., \& Santos, M. G. 2014, arXiv:1405.1452, arXiv:1405.1452

Camera, S., Raccanelli, A., Bull, P., et al., 2015, "Cosmology on the Largest Scales with the SKA", in proc. Advancing Astrophysics with the Square Kilometre Array, PoS(AASKA14)025

Camera, S., Santos, M. G., Bacon, D. J., et al. 2012, MNRAS, 427, 2079

Candès, E. 2006, Proceedings of the International Congress of Mathematicians, 3, 1433

Carrillo, R. E., McEwen, J. D., \& Wiaux, Y. 2014, MNRAS, 439, 3591

Corbel, S., Miller-Jones, J. C. A., Fender, R. P., et al., 2015, "Incoherent transient radio emission from stellar-mass compact objects in the SKA era", in proc. Advancing Astrophysics with the Square Kilometre Array, PoS(AASKA14)053

Donoho, D. 2006, IEEE Transactions on Information Theory, 52, 1289

Fabian, A. C. 2012, ARA\&A, 50, 455

Fender, R. et al., 2015, "The Transient Universe with the Square Kilometre Array", in proc. Advancing Astrophysics with the Square Kilometre Array, PoS(AASKA14)051

Fender, R. P., \& Bell, M. E. 2011, Bulletin of the Astronomical Society of India, 39, 315

Feroz, F., Hobson, M. P., \& Bridges, M. 2009, MNRAS, 398, 1601

Hojjati, A., Zhao, G.-B., Pogosian, L., et al. 2012, PhysRevD, 85, 043508

Ivezic, Z., Tyson, J. A., Acosta, E., et al. 2008, arXiv:0805.2366, arXiv:0805.2366

Jarvis, M., et al. 2015, "Cosmology with SKA Radio Continuum Surveys", in proc. Advancing Astrophysics with the Square Kilometre Array, PoS(AASKA14)018

Jarvis, M. J., Bonfield, D. G., Bruce, V. A., et al. 2013, MNRAS, 428, 1281

Kaiser, N. 1987, MNRAS, 227, 1

Kirk, D., Laszlo, I., Bridle, S., \& Bean, R. 2013, MNRAS, 430, 197

Lewis, A., \& Bridle, S. 2002, PhysRevD, 66, 103511

Lidz, A., Zahn, O., Furlanetto, S. R., et al. 2009, The Astrophysical Journal, 690, 252

Macquart, J., Keane, E. F., Grainge, K., et al., 2015, "Fast Transients at Cosmological Distances with the SKA", in proc. Advancing Astrophysics with the Square Kilometre Array, PoS(AASKA14)055

Marshall, P., Blandford, R., \& Sako, M. 2005, New Ast. Rev., 49, 387

McAlpine, M., Prandoni, I., Jarvis, M., et al., 2015, "The SKA view of the Interplay between SF and AGN Activity, and its role in Galaxy Evolution", in proc. Advancing Astrophysics with the Square Kilometre Array, PoS(AASKA14)083

McCracken, H. J., Milvang-Jensen, B., Dunlop, J., et al. 2012, A\&A, 544, A156

McDonald, P., \& Seljak, U. 2009, JCAP, 10, 7

McKean, J., Jackson, N., Vegetti, S., et al., 2015, "Strong gravitational lensing with the SKA", in proc. Advancing Astrophysics with the Square Kilometre Array, PoS(AASKA14)084

McMahon, R. G., Banerji, M., Gonzalez, E., et al. 2013, The Messenger, 154, 35

McQuinn, M., \& White, M. 2013, MNRAS, 433, 2857

Mellema, G., Koopmans, L. V. E., Abdalla, F. A., et al. 2013, Experimental Astronomy, 36, 235

Morales, M. F. 2006, ApJLett, 650, L21

Newman, J. A. 2008, ApJ, 684, 88

Oguri, M., \& Marshall, P. J. 2010, MNRAS, 405, 2579

Patel, P., Bacon, D. J., Beswick, R. J., Muxlow, T. W. B., \& Hoyle, B. 2010, MNRAS, 401, 2572

Pentericci, L. 2012, Memorie della Societa Astronomica Italiana, 83, 1123 
Pourtsidou, A., \& Metcalf, R. B. 2014, MNRAS, 439, L36

Raccanelli, A., Bull, P., Camera, S., et al., 2015, "Measuring redshift-space distortions with future SKA surveys", in proc. Advancing Astrophysics with the Square Kilometre Array, PoS(AASKA14)031

Raccanelli, A., Doré, O., Bacon, D. J., et al. 2014, arXiv:1406.0010, arXiv:1406.0010

Santos, M. et al., 2014b, Proceedings of "Advancing Astrophysics with the Square Kilometre Array", PoS(AASKA14)021

Santos, M., Bull, P., Alonso, D., et al., 2015, "Cosmology with a SKA HI intensity mapping survey", in proc. Advancing Astrophysics with the Square Kilometre Array, PoS(AASKA14)019

Skrutskie, M. F., Cutri, R. M., Stiening, R., et al. 2006, AJ, 131, 1163

Smolčić, V., Padovani, P., Delhaize, J., et al., 2015, "Exploring AGN Activity over Cosmic Time with the SKA", in proc. Advancing Astrophysics with the Square Kilometre Array, PoS(AASKA14)069

Stappers, B. W., Hessels, J. W. T., Alexov, A., et al. 2011, A\&A, 530, A80

Suyu, S. H., Marshall, P. J., Auger, M. W., et al. 2010, ApJ, 711, 201

Taylor, J. F., Ashdown, M. A. J., \& Hobson, M. P. 2008, MNRAS, 389, 1284

Wandelt, B. D., Larson, D. L., \& Lakshminarayanan, A. 2004, PhysRevD, 70, 083511

Wiersma, R. P. C., Ciardi, B., Thomas, R. M., et al. 2013, MNRAS, 432, 2615

Wright, E. L., Eisenhardt, P. R. M., Mainzer, A. K., et al. 2010, AJ, 140, 1868

Zhao, G.-B., Bacon, D., Maartens, R., et al., 2015, "Model-independent constraints on dark energy and modified gravity with the SKA", in proc. Advancing Astrophysics with the Square Kilometre Array, PoS(AASKA14) 165 